\title{
LA EVIDENCIA ACERCA DE LA CONTROVERSIA DE LAS VACUNAS QUE CONTIENEN TIMEROSAL Y SU ASOCIACIÓN CON EL AUTISMO*
}

\author{
Lisset García-Fernández¹,a, Adrián V. Hernández ${ }^{1,2, b}$, Víctor Suárez Moreno ${ }^{1, c}$, Fabián Fiestas ${ }^{1, d}$
}

RESUMEN

La vacunación es una de las medidas de mayor impacto en la salud pública para la reducción de la morbimortalidad infantil. El timerosal es un compuesto orgánico del mercurio utilizado como preservante de los frascos multidosis. Eventualmente, en el Perú, surgen olas de controversia acerca de la seguridad de estas vacunas, asociándolas especialmente con el autismo. Como resultado de estas controversias, se han propuesto, incluso, leyes que prohíben este tipo de vacunas, lo que tendría un importante impacto en los costos y en los aspectos logísticos de la estrategia nacional de vacunación. En este artículo se revisa la literatura sobre las principales controversias acerca de las vacunas que contienen timerosal y su supuesta asociación con el autismo. Se realiza una aproximación histórica sobre estas controversias, se hace una actualización de la evidencia científica disponible al momento, y se revisa la posición de los organismos internacionales más importantes con respecto a este tema. Se concluye que la evidencia científica no apoya la noción que exista una asociación entre el uso del timerosal en las vacunas con los trastornos del espectro autista en niños.

Palabras clave: Vacunas; Timerosal; Trastorno Autístico (fuente: DeCS BIREME)

\section{ADDRESSING THE CONTROVERSY REGARDING THE ASSOCIATION BETWEEN THIMEROSAL-CONTAINING VACCINES AND AUTISM}

\begin{abstract}
Vaccination is one of the most important public health interventions in the reduction childhood morbidity and mortality. Thimerosal is an organic mercury compound used as preservante in multi-dose vials. Often in Peru, there are waves of controversy about the safety of this type of vaccines, mainly arguing that there is an association between them and autism. As a result of these controversies, there have been some voices asking for laws banning thimerosal-containing vaccines, which would have a large impact in costs and the logistic aspects of the public vaccination programs. The aim of this article is to review the literature for the main controversies about thimerosal in vaccines and its supposed association to autism. We made an historical review about these controversies given the available scientific evidence and the statements from important international organizations. We concluded that the current available evidence do not support an association between thimerosal and childhood neurodevelopmental disorders, such as autism.
\end{abstract}

Key words: Vaccines; Thimerosal; Autistic disorder (source: MeSH NLM).

\section{INTRODUCCIÓN}

Las vacunas han demostrado ser una de las principales medidas de salud pública para disminuir la morbimortalidad infantil. Así, el porcentaje de disminución de las enfermedades inmunoprevenibles entre el siglo XX y el siglo XXI alcanza hasta el $100 \%$ en algunas enfermedades como la viruela, la difteria y la polio ${ }^{(1)}$.
La seguridad de las vacunas está garantizada por los diversos sistemas de vigilancia que se han implementado en los países que aplican regularmente las vacunas en los niños, y que señalan que los riesgos potenciales de las vacunas son muy escasos, siendo superados en gran medida, por los beneficios que se obtienen al aplicarlas $^{(2)}$.

\footnotetext{
Unidad de Análisis y Generación de Evidencias en Salud Pública (UNAGESP), Instituto Nacional de Salud. Lima, Perú.

Department of Quantitative Health Sciences, Lerner Research Institute, Cleveland Clinic, Cleveland. Ohio, EE. UU.

a Médica infectóloga; ${ }^{\mathrm{b}}$ médico epidemiólogo clínico, $\mathrm{PhD}$ in Health Science; ${ }^{\mathrm{c}}$ médico infectólogo, MPH in Public Health; ${ }^{\mathrm{d}}$ médico epidemiólogo

* Versión preliminar publicada como nota técnica 2012-4: "Efectos en la salud de las vacunas que contienen timerosal". Disponinble en: www.ins.gob.pe/ portal/jerarquia/4/825/publicaciones-unagesp/jer.825

Recibido: 12-04-13 Aprobado: 22-05-13
}

Citar como: García-Fernández L, Hernández AV, Suárez Moreno V, Fiestas F. La evidencia acerca de la controversia de las vacunas que contienen timerosal y su asociación con el autismo. Rev Peru Med Exp Salud Publica. 2013;30(2):268-74. 
Uno de los componentes de las vacunas es el timerosal. El timerosal está compuesto en un $49,6 \%$ de etilmercurio $(\mathrm{EtHg})$, compuesto orgánico del mercurio, y ácido tiosalicílico. El timerosal se encuentra en las vacunas a una concentración que varía de 0,003 a 0,01\% (entre $12,5 \mu \mathrm{g}$ y $50 \mu \mathrm{g}$ por dosis de vacuna que equivalen a una concentración entre 6,25 y $25 \mu$ g etilmercurio) ${ }^{(3,31)}$. Este compuesto ha sido ampliamente utilizado por la industria farmacéutica por sus propiedades bactericidas y antifúngicas en preparaciones como soluciones tópicas, ungüentos, sprays nasales, soluciones oculares, espermicidas vaginales $y$, principalmente, como preservante en viales multidosis de vacunas y otros productos biológicos inyectables como son los preparados de inmunoglobulinas ${ }^{(4)}$.

El beneficio de adicionar un preservante, como el timerosal, a los frascos de las vacunas multidosis se hizo evidente en estudios donde se vio que existe alto riesgo de contaminación bacteriana en frascos de este tipo de vacunas si no contienen ningún preservante. Este hecho puede causar síntomas graves e incluso muerte de los receptores. Como ocurrió en 1916, cuando cuatro niños murieron y 60 sufrieron síntomas graves debido a la contaminación con Staphylococcus aureus de un frasco de vacuna contra la tifoidea; y en 1928, en Australia, cuando esta misma bacteria contaminó un frasco con vacuna contra la difteria, causando la muerte de 12 de 21 niños ${ }^{(5)}$. Debido a esto, la Organización Mundial de la Salud (OMS) ha señalado que de ser retirado el timerosal de las vacunas, se pondría en riesgo la vida de las personas debido a la contaminación de los viales multidosis, estos permiten la implementación de programas de vacunación extendidos a nivel nacional ${ }^{(6)}$.

La seguridad de las vacunas que contienen timerosal ha sido respaldada por la OMS, mediante un reporte presentado en junio de 2012 realizado por el Comité Consultivo de Expertos en Inmunizaciones (SAGE, por sus siglas en inglés) ${ }^{(7,8)}$ donde se señala que, según la revisión de la literatura y la evidencia acumulada, apoyan la seguridad del uso del timerosal como preservante en las vacunas. Asimismo, agencias como la Academia Americana de Pediatría, en el 2013, ha señalado su posición a favor de mantener el timerosal en las vacunas ${ }^{(9)}$, retirando inclusive la declaración de 1999 donde se señalaba la medida precautoria con respecto a este compuesto en las vacunas ${ }^{(10)}$.

Sin embargo, el timerosal en las vacunas no ha estado libre de controversias, y algunos lo han señalado como causa de trastornos del espectro de autismo, especialmente en base a estudios en células, estudios en modelos animales o de extrapolaciones de evidencia de toxicidad de otros componentes que contienen mercurio, como el metilmercurio (11). En el presente artículo es revisan cuáles han sido estas controversias $y$ en qué se sustentan. Asimismo, se hace una actualización de la evidencia científica que evalúa la hipótesis que el timerosal es causa de un aumento de casos de trastornos del neurodesarrollo en niños.

\section{RECUENTO HISTÓRICO}

La asociación entre problemas del neurodesarrollo y las vacunas no es un tema nuevo ni exclusivo para las vacunas que contienen timerosal. Por ejemplo, un estudio publicado por Wakefield et al. en $1998{ }^{(12)}$ asoció a la vacuna contra sarampión, paperas y rubeola (SPR o MMR, por sus siglas en Inglés), la cual no contiene timerosal, con autismo. Específicamente, este autor describió 12 niños con alteraciones del desarrollo asociadas a síntomas gastrointestinales y regresión en el desarrollo, asociando el inicio de estos problemas luego a la vacunación con SPR según el reporte de los padres de familia. Si bien la vacuna SPR no contiene timerosal, este fue el inicio de una serie de hipótesis que apuntaban a una posible asociación de la vacunación con el autismo. Posteriormente, se demostró que la información descrita por Wakefield fue fraudulenta y la revista The Lancet tuvo que retirar el artículo. Estudios posteriores no han encontrado asociación entre esta vacuna y el autismo ${ }^{(13,14)}$.

Luego de lo publicado por Wakefield en 1998, la sociedad científica y la población americana quedó muy sensibilizada y preocupada, y surgieron, además, muchas presiones políticas. Por lo que la Administración de Medicamentos y Alimentos de EE. UU. (Food and Drug Administration, FDA) solicitó a los fabricantes de medicamentos, entre ellos a los fabricantes de vacunas, la información sobre el contenido de timerosal en sus preparados, dicha respuesta por parte de la industria se dio en diciembre de 1998 y abril de $1999{ }^{(15)}$. Tras este llamado, se generó alarma sobre los daños potenciales del timerosal en las vacunas y, en la segunda mitad de 1999, tanto los Estados Unidos de América, a través de la Academia Americana de Pediatría (AAP), el Servicio de Salud Pública de los Estados Unidos (USPHS) así como la Unión Europea, por medio de la Agencia Europea para la Evaluación de Productos Medicinales (EMEA), publicaron su posición con respecto a la presencia del timerosal en las vacunas. Ellos señalaron que a pesar de no haber evidencia clara sobre la asociación entre las vacunas que contienen timerosal y problemas potenciales en la salud, ante la controversia y sensibilidad de la población, recomendaban el retiro del timerosal de las vacunas como medida de precaución. Sin embargo, recomendaron la revisión de 
los datos y de los estudios existentes en dicho momento, además de la realización de mayores estudios que permitan establecer o rechazar esta asociación con mayor solidez ${ }^{(15,16)}$. Luego de casi 15 años de dicha recomendación, se ha producido mucha nueva evidencia científica, usando metodologías rigurosas, que permite ahora a la comunidad científica hacer una mejor evaluación de la controversia y tener un asidero más sólido a la hora de hacer recomendaciones en el tema.

\section{LA EVIDENCIA CIENTÍFICA ACTUAL RESPECTO A LAS CONTROVERSIAS}

En medicina, la evidencia científica se ordena de manera jerárquica según la solidez con que los datos sostienen una pieza de conocimiento. En este sentido, las revisiones sistemáticas están entre los niveles más altos de evidencia científica. Así, en el 2004 se publicó una revisión sistemática, realizada por Parker et al. (17), la cual incluyó doce estudios primarios identificados y calificados usando una metodología rigurosa. Esta revisión sistemática no encontró evidencia de asociación entre las vacunas que contienen timerosal y trastornos del espectro autista. Además, esta revisión encontró que aquellos estudios que sí mostraban una asociación tenían serios problemas metodológicos que invalidaban sus hallazgos. Es así que, concluyeron que no existe evidencia científica de una relación entre las vacunas que contienen timerosal y los trastornos del espectro autista, por lo que no se justificarían cambios en los esquemas que contengan vacunas con timerosal a otros esquemas con vacunas sin timerosal.

Luego de esta revisión sistemática, se han realizado otros estudios, igual de rigurosos, que han señalado hallazgos similares, consistentes con la hipótesis de que los trastornos del espectro autístico son eventos independientes, y no relacionados, con las vacunas que contienen timerosal. En 2007 Thompson et al. (18) realizaron un estudio de 1047 niños de 7 a 10 años a los que evaluaron por 42 eventos neuropsiquiátricos usando instrumentos estandarizados y de los que se obtuvo información cuidadosa y exhaustiva acerca de su exposición a mercurio proveniente del timerosal durante la fase prenatal, perinatal y hasta los 7 meses de edad. Los autores concluyeron que no pudieron encontrar indicios consistentes de que el timerosal causara deficiencias en el desarrollo o funcionamiento neuropsicológico luego de varios años de exposición.

Por su parte, en el 2009, Tozzi et al.(19) realizaron un estudio de cohortes en niños que habían participado de un ensayo clínico durante el primer año de vida y en el que habían sido aleatorizados de tal manera que un grupo recibiera vacunas que hacían que la dosis acumulada de timerosal sea de $62,5 \mu \mathrm{g}$ y otro grupo a dosis de $137,5 \mu \mathrm{g}$. Estos dos grupos de niños fueron comparados entre sí respecto a 24 eventos neuropsicológicos, evaluados mediante instrumentos estandarizados a los 10 años de haber recibido las vacunas. No hubo asociaciones entre las dosis acumuladas de timerosal y los eventos neuropsicológicos evaluados, excepto en dos de ellos (el Finger-tapping test, que sirve para evaluar la velocidad y el control motor, y el Boston naming test, utilizado para evaluar el recuerdo de palabras), aunque estas asociaciones fueron tan pequeñas que, además de ser estadísticamente muy inestables, no se puede determinar con claridad su implicancia clínica. Por lo que, en general, los autores concluyen que su estudio apoya la noción de que el timerosal en las vacunas no está asociado a un mayor riesgo de deficiencias neuropsicológicas en los niños.

En el 2010, Price et al. ${ }^{(20)}$ informaron hallazgos similares en un estudio caso-control con aproximadamente 1000 niños. En este estudio, 256 niños con algún trastorno del espectro autístico (evaluados con instrumentos estandarizados) fueron comparados con 752 niños sin evidencia de tener este tipo de trastornos. La exposición a timerosal a través de vacunas fue evaluada usando registros electrónicos de inmunizaciones, historias clínicas y entrevista a los padres. Los resultados mostraron que la exposición acumulada de timerosal del nacimiento al mes de edad, del nacimiento a los 7 meses, o del nacimiento a los 20 meses de edad no estuvieron asociadas a ninguna categoría del espectro autístico, siendo ello nuevamente consistente con la noción de que el timerosal no causa problemas del espectro del autismo.

Se han hecho estudios ecológicos tratando de establecer la asociación entre timerosal y trastornos del autismo (21). Uno de estos estudios fue realizado con información proveniente de California y fue presentado en una reunión del Comité de Seguridad de las Inmunizaciones del Instituto de Medicina de los Estados Unidos (IOM) en julio de 2001. En este estudio se comparó la dosis acumulada promedio de exposición al timerosal con la incidencia del autismo por 10000 personas-año. Se observó que los casos de problemas del neurodesarrollo aumentaron de manera más rápida a partir del año 1990 coincidiendo con un aumento en el uso de vacunas que contienen timerosal. Con esto, los autores concluyeron que hay una asociación entre los problemas neurológicos asociados con el autismo y el aumento de las dosis acumulativas de timerosal. Sin embargo, existen muchas limitaciones en esta investigación, ya que no toma en cuenta otros factores 
que pudieron haber influido en sus resultados, como son otras fuentes de contaminación por mercurio en el ambiente, otros factores diferentes al mercurio que han sido asociados a trastornos del espectro autista, y a la evidencia que ya los casos de autismo venían aumentando en años anteriores al aumento de las vacunas conteniendo timerosal y anteriores a la mayor cobertura de vacunación.

Cuando se comparó la investigación proveniente de California con la información proveniente de Suecia y Dinamarca ${ }^{(21)}$, donde los sistemas de registros de salud son rigurosos y extensivos, se observó que el aumento de los casos de autismo durante la década de los ochenta se mantuvo durante la década de los noventa, a pesar de que en ambos países las vacunas con timerosal habían sido retiradas en el año 1992 (Figura 1). Esta evidencia contradice fuertemente la noción que las vacunas con timerosal causan autismo. Una de las explicaciones que se ha dado para la coincidencia entre el aumento del uso del timerosal y el aumento simultaneo en la incidencia de autismo, como fue sugerido en el estudio de California, es mayor conocimiento conciencia acerca del autismo por parte de la comunidad médica, debido al cual estos empezaron a diagnosticar más casos de este trastorno. Tampoco se puede descartar que otros factores ambientales o genéticos, aún no identificados, hayan incrementado el número de niños autistas.

En este sentido, se debe considerar otras posibles causales de las enfermedades del espectro del autismo. Al respecto existen varios estudios que han evaluado diversas hipótesis como alteraciones cromosómicas, problemas genéticos, enfermedades físicas o mentales de la madre, anomalías orgánicas o funcionales del cerebro, enfermedades sistémicas (como enfermedades autoinmunes, alteraciones hormonales, trastornos electrolíticos, estrés oxidativo, entre otras) ${ }^{(22)}$, inclusive se ha encontrado evidencia que el antecedente que madre haya sido víctima de abuso infantil está asociado con un mayor riesgo que esta tenga descendencia con autismo ${ }^{(23)}$.

Por otro lado, el IOM publicó un libro que contiene una amplia revisión con respecto a la seguridad de las inmunizaciones, señalando entre sus conclusiones que la evidencia existente rechaza una relación causal entre las vacunas que contienen timerosal y el autismo ${ }^{(14)}$.

Quizá una de las razones por las que se ha relacionado el timerosal (etilmercurio) con alteraciones neurológicas y del neurodesarrollo es que existe confusión entre lo que es el etilmercurio y el metilmercurio, otro compuesto orgánico del mercurio ${ }^{(7,24)}$. El metilmercurio se encuentra en la naturaleza, como en algunos pescados, y se utiliza además en la amalgama para las curaciones dentales, pero que no se usa para las vacunas. Debido a cierta evidencia que asocia el metilmercurio con problemas con el neurodesarrollo, se han establecido diferentes límites de exposición acumulativa para niños interpuestas por agencias como la FDA de los EE. UU., la Organización Mundial de la Salud y la Agencia de Protección Ambiental, entre otras, basados en la farmacocinética del metilmercurio en adultos ${ }^{(25)}$. Como consecuencia de que se toman ambos compuestos como los mismos (etilmercurio y el metilmercurio), entonces los límites establecidos para el metilmercurio han sido extrapolados a la acumulación del etilmercurio en niños, aunque esta extrapolación aún no tiene evidencia que la justifique científicamente. De hecho, no se ha establecido la equivalencia del metabolismo entre el etilmercurio y
A

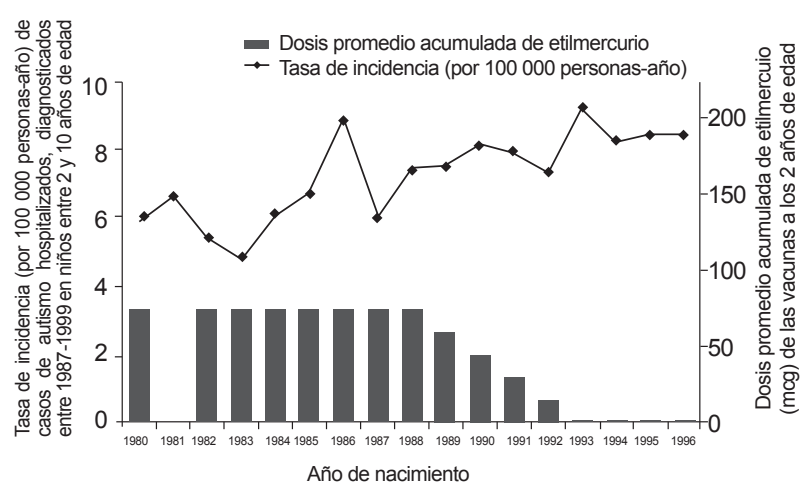

B

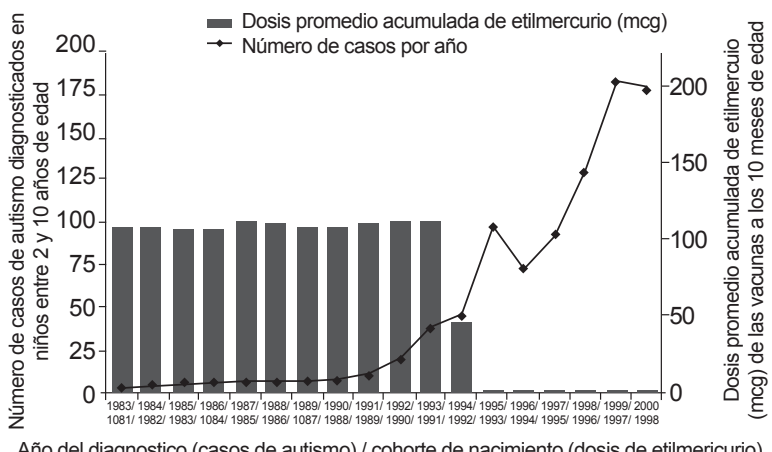

Año del diagnostico (casos de autismo) / cohorte de nacimiento (dosis de etilmericurio)

Figura 1. Estudios ecológicos que muestran la relación entre la dosis acumulada de etilmercurio y el número de casos de autismo

A. Análisis ecológico en niños suecos comparando la dosis acumulada de etilmercurio y el número de casos de autismo. Luego del retiro de etilmercurio, el número de casos de autismo se incrementó. B. Análisis ecológico en niños daneses comparando la dosis acumulada de etilmercurio y el número de casos de autismo. Luego del retiro del etilmercurio el número de casos de autismo se incrementó. (Reimpreso de American Journal of Preventive Medicine; 25(2); Stehr-Green P, Tull P, Stellfeld M, Mortenson P-B, Simpson D; Autism and thimerosal-containing vaccines; pp. 101-6; 2003; con permiso de Elsevier). 
el metilmercurio mediante estudios farmacocinéticos o toxicológicos en humanos ${ }^{(7)}$. Esto se explica debido a que la cinética de cada uno de los compuestos (es decir su metabolismo de acuerdo con su composición química) determina su toxicidad (4), existe evidencia que el etilmercurio se degrada más rápidamente que el metilmercurio ${ }^{(26)}$. Por ejemplo, estudios en modelos animales han permitido observar que el pasaje del metilmercurio a través de la barrera hematoencefálica es facilitado por un mecanismo de transporte activo, mientras que para el etilmercurio este pasaje más bien es entorpecido por su mayor tamaño molecular y su descomposición más rápida. Por lo tanto, la evidencia indica que los riesgos que se atribuyen al metilmercurio (que no está en las vacunas) sobreestiman el riesgo que existe con relación al etilmercurio, que es el compuesto de las vacunas ${ }^{(27)}$

Existen estudios que han evaluado la farmacocinética del etilmercurio proveniente de las vacunas con timerosal. Ellos han señalado que este es metabolizado y excretado del organismo, por lo que no se acumula en ningún tejido. Un estudio en niños comparó la concentración de mercurio en sangre, orina y heces en un grupo que recibió vacunas con timerosal frente a un grupo que recibió vacunas sin este compuesto, y encontró que las dosis de mercurio en los fluidos estudiados se encontraba muy por debajo de los límites establecidos para el metilmercurio para el grupo que recibió vacunas con timerosal; mientras que en el grupo que recibió vacunas sin timerosal, todos, excepto uno tuvieron niveles indetectables de mercurio. Además sugirió que la vida media del etilmercurio sería mucho más corta que la del metilmercurio según los cálculos realizados (7 días, IC 95\% 4-10) ${ }^{(28)}$. Otro estudio, diseñado para evaluar la farmacocinética del timerosal en recién nacidos y en niños de 2 y 6 meses de edad, estimó que la vida media del etilmercurio fue de 3,7 días (IC 95\% Cl: 2,9 - 4,5), llegando a niveles prevacunales en menos de un mes ${ }^{(29)}$. Se han realizado estudios similares en otras poblaciones como prematuros y recién nacidos de bajo peso en los que la vida media del etilmercurio fue calculada en 6,3 días (IC 95\%: 3,9$8,8)$ y los niveles de mercurio regresaron a los valores

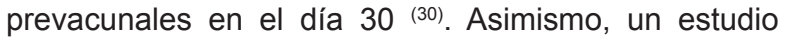
realizado en mujeres adultas sometidas a tratamiento con la vacuna de toxoide estafilocócico para tratamiento de fibromialgia ( $1 \mathrm{~mL}$ de vacuna cada 3-4 semanas por lo menos un año; cada vacuna con $0,01 \%$ de timerosal), encontró que luego de 2 a 4 semanas los niveles de etilmercurio retornaron al basal, situación similar a las pacientes no tratadas ${ }^{(31)}$.

Se ha afirmado también que las dosis de etilmercurio al final de todo el periodo de vacunación alcanzan niveles acumulativos que sobrepasan los niveles establecidos, no para el etilmercurio, sino para el metilmercurio; sin embargo, las vacunas son administradas según un esquema detallado que comprende la vacunación desde el nacimiento hasta los cuatro años de vida con períodos espaciados entre cada vacuna como mínimo de 2 meses ${ }^{(32)}$. Por ello, no es acertado señalar que el mercurio proveniente de las vacunas en los niños permanece acumulado hasta el final del periodo de vacunación, ya que como hemos visto en el párrafo anterior, la evidencia señala que, según la farmacocinética de este elemento, se depura de forma completa en menos de un mes ${ }^{(28-31)}$. Asimismo, el modelo farmacocinético utilizado en estos estudios corresponde a una distribución unicompartamental, lo cual se ajusta a las sustancias que no tienen la propiedad de depositarse en los tejidos ${ }^{(28,29)}$. Por el contrario, al ser explorado un modelo bicompartamental (modelo que siguen las sustancias que se depositan en el organismo) se ha observado que los datos no se ajustan al metabolismo del timerosal que se evidencia en niños ${ }^{(29)}$. Por lo tanto, la evidencia científica existente sostiene que el timerosal no se acumula en los tejidos del cuerpo humano.

\section{EVALUANDO LA ALTERNATIVA DE VACUNAS SIN TIMEROSAL}

Las implicancias de cambiar los esquemas que usan vacunas que contienen timerosal a un esquema que use vacunas sin este compuesto tendrían gran impacto tanto en la producción como en la logística de los programas de vacunación, sin mencionar el gran incremento en los costos no solo para países de medianos y bajos ingresos, como el Perú, sino también en los costos a nivel internacional ${ }^{(8,23)}$. Con respecto al primer punto, si se decidiera cambiar a vacunas sin timerosal, se dejaría de adquirir vacunas multidosis para comprar vacunas monodosis -que son las que no contienen este preservante-incrementando la demanda en la producción de estas últimas y por lo tanto los costos, inclusive con el riesgo de sobrepasar la capacidad de respuesta de los fabricantes ${ }^{(9)}$. Por otro lado, el contar con vacunas en frascos monodosis sobrepasaría en gran proporción la capacidad de las cadenas de frío en los establecimientos de salud a nivel nacional, lo que aumentaría los costos en la adquisición de equipos en número y tamaño, y de hecho en las regiones más pobres sería imposible de implementar. Así, la Organización Panamericana de la Salud y el Fondo de las Naciones Unidas para la Infancia (UNICEF), han hecho estimados de los costos que implicarían estos cambios, los cuales llegarían a cifras mayores a los 300 millones de dólares ${ }^{(33)}$.

Se sabe que existen actualmente otros compuestos que servirían como preservantes que están siendo 
evaluados para reemplazar al timerosal, sin embargo, el tiempo necesario para su comercialización y licencia es prolongado, sin contar con que aún son necesarios más ensayos clínicos para evaluar no solo la estabilidad de las vacunas en las que sean utilizados estos nuevos preservantes, sino también la seguridad y eficacia que le otorgarían a las vacunas ${ }^{(6,34,35)}$.

\section{CONCLUSIONES}

La evidencia utilizada para sugerir que las vacunas que contienen timerosal producen autismo o enfermedades del neurodesarrollo infantil no surge de estudios adecuados para evaluar asociación o causalidad. La mayoría de estos estudios han sido realizados en células, en modelos animales o de extrapolaciones de evidencia de toxicidad del metilmercurio. Por otro lado, existe una sólida evidencia, proveniente de estudios de mayor rigurosidad y calidad, que contradice la posibilidad asociación causal entre las vacunas que contienen etilmercurio y enfermedades del neurodesarrollo infantil, como son los trastornos del espectro del autismo.

Así, como la mejor evidencia científica existente es consistente con la noción de que las vacunas que contienen timerosal no están asociadas con los trastornos del espectro del autismo, entonces no se justificaría hacer cambios en los esquemas actuales de vacunación para introducir esquemas que no contengan vacunas con timerosal. De hacerlo, se podría más bien incurrir en problemas de aumento de costos y de limitaciones logísticas que afectarían seriamente la cobertura de los programas nacionales o públicos de vacunación, especialmente para las regiones más pobres. El hacerlo significaría un riesgo que es demasiado alto comparado con el beneficio incierto que se obtendría, ya que no es posible calcular cuál sería la reducción en la incidencia de trastornos del autismo a nivel poblacional si se retiraran las vacunas que contienen timerosal, y por el contrario, la evidencia de varios países muestra que la incidencia de autismo ha aumentado sostenidamente aun después de haber retirado las vacunas que contienen timerosal, en gran medida, de los esquemas de vacunación.

Contribuciones de autoría: LG, AH FF y VS participaron en la concepción, diseño y revisión del artículo. LG escribió el primer borrador del artículo. AH y LG hicieron la búsqueda, recolección y evaluación de la literatura. Todos los autores aprueban la versión a publicar.

Fuentes de financiamiento: Instituto Nacional de Salud

Conflictos de interés: los autores declaran no tener conflictos de interés

\section{REFERENCIAS BIBLIOGRÁFICAS}

1. Centers for Disease Control and Prevention. Appendix G: Data and Statistics - Impact of Vaccines in the 20th and 21st Centuries. In: Epidemiology and Prevention of Vaccine-Preventable Diseases. 12th ed. Washington, DC: Public Health Foundation; 2012. pg. G-7.

2. Roush SW, Murphy TV; VaccinePreventable Disease Table Working Group. Historical comparisons of morbidity and mortality for vaccinepreventable diseases in the United States. JAMA. 2007;298(18):2155-63.

3. Ball LK, Ball R, Pratt RD. An assessment of thimerosal use in childhood vaccines. Pediatrics. 2001;107(5):1147-54.

4. Risher JF, Murray HE, Prince GR. Organic mercury compounds: human exposure and its relevance to public health. Toxicol Ind Health. 2002;18(3):109-60.

5. Wilson G. The Hazards of Immunization. New York: The Athlone Press; 1967.
6. World Health Organization. Thiomersal as a vaccine preservative. Weekly Epidemiological Record. 2000;75:12-6.

7. World Health Organization. Global advisory Committee on Vaccine Safety, June 2012. Weekly Epidemiological Record. 2012;30(87):281-2.

8. World Health Organization. Meeting of the Strategic Advisory Group of Experts on Immunization, November 2012 conclusions and recommendations. Weekly Epidemiological Record. 2013;88(1):1-16.

9. Orenstein WA, Paulson JA, Brady MT, Cooper LZ, Seib K. Global vaccination recommendations and thimerosal. Pediatrics. 2013;131(1):149-51.

10. Policy Statement--AAP Publications Reaffirmed and Retired. Pediatrics. 2010 Jul 1;126(1):177.

11. Maya L, Luna F. El timerosal y las enfermedades del neurodesarrollo infantil. An Fac med. 2006;67(3):255-74.
12. Wakefield AJ, Murch SH, Anthony A, Linnell J, Casson DM, Malik M, et al. Ileal-lymphoid-nodular hyperplasia, non-specific colitis, and pervasive developmental disorder in children. Lancet. 1998;351(9103):637-41.

13. Wilson K, Mills E, Ross C, McGowan J, Jadad A. Association of autistic spectrum disorder and the measles, mumps, and rubella vaccine: A systematic review of current epidemiological evidence. Arch Pediatr Adolesc Med. 2003;157(7):628-34.

14. Immunization Safety Review Committee. Immunization Safety Review: Vaccines and Autism. Washington, DC: The National Academies Press; 2004.

15. Joint Statement of the American Academy of Pediatrics (AAP) and the United States Public Health Service (USPHS). Pediatrics. 1999; 104(3):568-9.

16. The European Agency for the Evaluation of Medicinal Products, 
Human Medicines Evaluation Unit. EMEA public statement on thiomersal containing medicinal products [Internet]. London: EMEA; 1999 [citado el 15 de nero del 2013]. Disponible en: http://www.ema. europa.eu/docs/en_GB/document_ library/Scientific_guideline/2009/09/ WC500003902.pdf

17. Parker SK, Schwartz B, Todd J, Pickering LK. Thimerosal-containing vaccines and autistic spectrum disorder: a critical review of published original data. Pediatrics. 2004;114(3):793-804.

18. Thompson WW, Price C, Goodson B, Shay DK, Benson P, Hinrichsen VL, et al. Early thimerosal exposure and neuropsychological outcomes at 7 to 10 years. $\mathrm{N}$ Engl J Med. 2007;357(13):1281-92.

19. Tozzi AE, Bisiacchi P, Tarantino V, Mei BD, D'Elia L, Chiarotti F, et al. Neuropsychological performance 10 years after immunization in infancy with thimerosal-containing vaccines. Pediatrics. 2009;123(2):475-82.

20. Price CS, Thompson WW, Goodson B, Weintraub ES, Croen LA, Hinrichsen VL, et al. Prenatal and infant exposure to thimerosal from vaccines and immunoglobulins and risk of autism. Pediatrics. 2010;126(4):656-64.

21. Stehr-Green P, Tull P, Stellfeld M, Mortenson PB, Simpson D. Autism and thimerosal-containing vaccines: lack of consistent evidence for an association. Am J Prev Med. 2003;25(2):101-6.

22. Hughes JR. A review of recent reports on autism: 1000 studies published in 2007. Epilepsy Behav. 2008;13(3):425-37.

23. Roberts AL, Lyal K, Rich-Edwards JW, Ascherio A, Weisskopf MG. Association of maternal exposure to childhood abuse with elevated risk for autism in offspring. JAMA Psychiatry. 2013;170(5):508-15.

24. The American Academy of Pediatrics. Ban on all mercury-based products would risk global immunization efforts, says AAP, WHO. AAP News [Internet]. 2012 Jun 1 [citado el 2 de abril del 2013]. Disponible en: http://aapnews.aappublications. org/content/early/2012/06/01/ aapnews.20120601-1

25. Bigham M, Copes R, Srour L. Exposure to thimerosal in vaccines used in Canadian infant immunization programs, with respect to risk of neurodevelopmental disorders. Can Commun Dis Rep. 2002;28(9):69-80.

26. Dórea JG, Farina M, Rocha JBT. Toxicity of ethylmercury (and Thimerosal): a comparison with methylmercury. J Appl Toxicol. 2013 Feb 11. doi: 10.1002/jat.2855. [Epub ahead of print]

27. Magos L. Review on the toxicity of ethylmercury, including its presence as a preservative in biological and pharmaceutical products. J Appl Toxicol. 2001;21(1):1-5.

28. Pichichero ME, Cernichiari E, Lopreiato J, Treanor J. Mercury concentrations and metabolism in infants receiving vaccines containing thiomersal: a descriptive study. Lancet. 2002;360(9347):1737-41.

29. Pichichero ME, Gentile A, Giglio N, Umido V, Clarkson T, Cernichiari E, et al. Mercury levels in newborns and infants after receipt of thimerosalcontaining vaccines. Pediatrics. 2008;121(2):e208-214.

30. Pichichero ME, Gentile A, Giglio N, Alonso MM, Fernandez Mentaberri MV, Zareba G, et al. Mercury levels in premature and low birth weight newborn infants after receipt of thimerosal-containing vaccines. J. Pediatr. 2009;155(4):495-9.

31. Barregard L, Rekić D, Horvat M, Elmberg L, Lundh T, Zachrisson O. Toxicokinetics of mercury after longterm repeated exposure to thimerosalcontaining vaccine. Toxicol Sci. 2011;120(2):499-506.

32. Perú, Ministerio de Salud. NTS N080MINSA/DGSP-V.02: "Norma Técnica de Salud que establece el Esquema Nacional de Vacunación". Lima: Ministerio de Salud; 2002.

33. King K, Paterson M, Green SK. Global justice and the proposed ban on thimerosal-containing vaccines. Pediatrics. 2013;131(1):154-6.

34. Ball R. Substituting Thimerosal Preservative used in Vaccines: FDA perspective [Internet]. Silver Spring: US Food and Drug Administration; 2012 [citado el 2 de abril del 2013]. Disponible en: http://who.int/ immunization/sage/meetings/2012/ april/USFDA_perspective_ thimerosal_alternatives.pdf

35. Bossche GV. Alternative Preservatives for Vaccines [Internet]. Geneva: WHO; 2012 [citado el 2 de abril del 2013]. Disponible en: http://who.int/ immunization/sage/meetings/2012/ april/alternative_preservatives_ vaccines_bossche.pdf

Correspondencia: Fabián Fiestas

Dirección: Jr. Huamachuco 1712, Jesús

María, Perú.

Teléfono: (511) 748000 Anexo 6651

Correo electrónico:fabianfiestas@yahoo.com 